\title{
Surveillance for Mass Gatherings: Super Bowl XLIX in Maricopa County, Arizona, 2015
}

\author{
Aurimar Ayala*, Vjollca Berisha and Kate Goodin \\ Public Health, Maricopa County, Phoenix, AZ, USA
}

\section{Objective}

To describe the enhanced epidemiologic surveillance efforts in place during Super Bowl XLIX and related events, review epidemiologic surveillance results, discuss novel approaches for near real-time surveillance for situational awareness and early event detection and examine lessons learned for surveillance strategies during mass gatherings.

\section{Introduction}

Super Bowl XLIX took place on February $1^{\text {st }}, 2015$ in Glendale, Arizona. In preparation for this large scale public event and related activities, the Maricopa County Department of Public Health (MCDPH) developed methods for enhanced surveillance, situational awareness and early detection of public health emergencies.

\section{Methods}

From July 2014 to January 2015, an Epidemiology Work Group and a Public Health and Medical Resource Work Group met on a monthly basis to develop and coordinate epidemiologic surveillance strategies with local, state and federal partners. Surveillance strategies were developed and coordinated to monitor levels of disease activity and provide situational awareness during Pro Bowl (January $25^{\text {th }}$, 2015), NFL Experience and Super Bowl Central (January 24 ${ }^{\text {th }}, 2015$ through February $1^{\text {st }}, 2015$ ) and Super Bowl. Fiesta Bowl (December $31^{\text {st }}$, 2015) was selected to pilot test surveillance strategies. Strategies included enhanced surveillance alerts, enhanced animal surveillance, field syndromic surveillance at first aid stations, syndromic surveillance for emergency room visits, hotel surveillance, urgent care surveillance, mortality surveillance, emergency medical services (EMS) surveillance, media surveillance and aberration detection algorithms for notifiable diseases.

\section{Results}

Surveillance strategies were successfully tested during Fiesta Bowl. During Super Bowl and associated events, field surveillance collected information on 4 distinct syndromes (gastrointestinal, respiratory, dermatological and neurological) as well as injuries. Real-time mapping of field surveillance data was also in place and aided in the evaluation of syndromes reported. Aberration detection algorithms were run daily to detect illness reported to Arizona's notifiable disease surveillance system. Enhanced surveillance alerts were sent to healthcare providers asking them to report any increases in illness or unusual illness presentation. Alerts were implemented from January 22th, 2015 to February 6, 2015. Enhanced animal surveillance was in place during all events. The Arizona Prehospital Information and EMS Registry System was utilized to monitor for gastrointestinal and respiratory syndromes. Influenza-like illness surveillance for outpatient sentinel sites was also in place. Poison Control Center data and newly developed algorithms for mortality surveillance within an all hazards approach were analyzed daily. MCDPH monitored foodborne outbreaks and produced a daily outbreak report. Syndromic surveillance in hotels and urgent cares located within a 5 mile radius of the events was conducted. In addition, the NFL clinic provided daily reports to MCDPH. The MCDPH Public Health Incident Command Center was activated and the Intelligence
Section, responsible for epidemiologic surveillance, produced daily Intelligence Section Report summarizing results from all surveillance efforts. Surveillance result highlights included influenza widespread activity, increased influenza activity reported from urgent cares and several influenza cases reported within the NFL clinic. In addition, an investigation into a cyanide single event exposure was investigated and determined not to be a public health threat. Field surveillance efforts documented minor injuries at all events and sporadic cases of gastrointestinal and neurological (mostly headaches) disease. Enhanced animal surveillance reports included a cat suspected for plague and tularemia and an investigation of highly pathogenic avian influenza in a backyard chicken flock. Laboratory results in both instances were negative. Aberration detection algorithms detected an increase in measles reports associated with Disneyland exposure and syndromic surveillance systems were used during this investigation successfully.

\section{Conclusions}

Coordinated enhanced epidemiologic surveillance during Super Bowl XLIX increased the response capacity and preparedness of the public health department to make informed decisions and take public health actions in a timely manner during this mass gathering event. MCDPH plans to continue development of novel tools and protocols for epidemiologic surveillance during mass gatherings to increase capacity for near real-time surveillance for situational awareness and early event detection.

\section{Keywords}

Syndromic Surveillance; Mass Gatherings; Situational Awareness

\section{*Aurimar Ayala}

E-mail: aurimarayala@mail.maricopa.gov 